Pesq. Vet. Bras. 37(11):1341-1344, novembro 2017 DOI: $10.1590 / \mathrm{S} 0100-736 \mathrm{X} 2017001100024$

\title{
Origem dos nervos do plexo braquial de sagui-de-tufos- brancos (Callithrix jacchus Linnaeus, 1758) ${ }^{1}$
}

\author{
Brunna M.R. Falcão², José R.S. Santos ${ }^{3}$, Ana Y.F. de La Salles ${ }^{2}$, Artur N. Carreiro², João \\ A.R.A. Diniz ${ }^{3}$, Rômulo F.F. Dias ${ }^{3}$, Danilo J.A. Menezes ${ }^{2,4 *}$ e Gildenor X. Medeiros ${ }^{3}$
}

\begin{abstract}
Falcão B.M.R., Santos J.R.S., De La Salles A.Y.F., Carreiro A.N., Diniz J.A.R.A., Dias R.F.F., Menezes D.J.A. \& Medeiros G.X. 2017. [Origin of brachial plexus nerves for common marmoset (Callithrix jacchus Linnaeus, 1758).] Origem dos nervos do plexo braquial de sagui-de-tufos-brancos (Callithrix jacchus Linnaeus, 1758). Pesquisa Veterinária Brasileira 37(11):1341-1344. Programa de Graduação em Medicina Veterinária, Universidade Federal de Campina Grande, Av. Universitária, Cx. Postal 64, Santa Cecília, Patos, PB 58708-110, Brazil. E-mail: mdanayres@gmail.com

The common marmoset (Callithrix jacchus) is a small primate that inhabits shrub forests of the Caatinga and the Atlantic Forest in Brazilian Northeast. This species of marmoset is very common and easy to adaptation in captivity, aspect that encourages illegal capture of these animals that are victims of mistreatments, causing injuries. The lack of knowledge in anatomy in marmosets and other wildlife hinders the safe application of clinical, surgical and therapeutic procedures. The brachial plexus has been described in some species of animals, but so far, there is no description for common marmoset (Callithrix jacchus). To study the brachial plexus of these animals were dissected ten corpses of common marmosets (Callithrix jacchus) adults, being five males and five females. In all animals, the brachial plexus was originated from spinal nerves C5, C6, C7, C8 and T1, with few variations in origin and the contributions of the nerves in both antimeres, forming three trunks: the cranial (C5 and C6), medium (C7) and caudal (C8 and T1).
\end{abstract}

INDEX TERMS: Brachial plexus nerves, common marmoset, Callithrix jacchus, Callitrichinae, anatomy, primates.

RESUMO.- 0 sagui-de-tufos-brancos (Callithrix jacchus) é um pequeno primata que habita florestas arbustivas da Caatinga e a Mata Atlântica do Nordeste brasileiro. Essa espécie de sagui é muito comum e de fácil adaptação ao cativeiro, aspecto que estimula a captura clandestina desses animais, os quais são vítimas de maus tratos que causam lesões. A

\footnotetext{
${ }^{1}$ Recebido em 13 de outubro de 2016.

Aceito para publicação em 6 de fevereiro de 2017.

${ }^{2}$ Programa de Pós-Graduação em Medicina Veterinária, Unidade Acadêmica de Medicina Veterinária (UAMV), Centro de Saúde e Tecnologia Rural (CSTR), Universidade Federal de Campina Grande (UFCG), Av. Universitária, Cx. Postal 64, Santa Cecília, Patos, PB 58708-110, Brasil. E-mail: brunnamrfalcao@hotmail.com, anayasha@hotmail.com, arturpets1992@gmail.com

${ }^{3}$ UAMV, CSTR-UFCG, Av. Universitária, Cx. Postal 64, Santa Cecília, Patos, PB 58708-110.E-mail: jromulosmedvet@gmail.com, joao-diniz15@hotmail.com, romuloffdias@gmail.com,mdanayres@gmail.com,gxmedeiros@ig.com.br

${ }^{4}$ Departamento de Morfologia, Centro de Biociências (CB), Universidade Federal do Rio Grande do Norte (UFRN), Campus Lagoa Nova, Cx. Postal 1524, Natal, RN 59078-970, Brasil. *Autor para correspondência: mdanayres@gmail.com
}

falta de conhecimento da anatomia de saguis e outros animais silvestres dificulta a aplicação segura de procedimentos clínicos, cirúrgicos e terapêuticos. 0 plexo braquial tem sido descrito em algumas espécies animais, porém até o momento não existia descrição em saguis-de-tufos-brancos (Callithrix jacchus). Para estudar o plexo branquial desses animais foram dissecados dez cadáveres de saguis-de-tufos-brancos (Callithrix jacchus) adultos sendo cinco machos e cinco fêmeas. Em todos os animais o plexo braquial originou- se dos nervos espinhais C5, C6, C7, C8 e T1, com poucas variações na origem e nas contribuições dos nervos, em ambos os antímeros, formando três troncos: cranial (C5 e C6), médio (C7) e caudal (C8 e T1).

TERMOS DE INDEXAÇÃO: Plexo braquial, sagui-de-tufos-brancos, Callithrix jacchus, Callitrichinae, anatomia, primatas.

\section{INTRODUÇÃo}

O sagui-de-tufos-brancos (Callithrix jacchus) pertencem a ordem Primates e a família Callitrichidae, grupo formado 
pelos menores primatas antropoides (Rylands 2012). Habita florestas arbustivas da Caatinga e a Mata Atlântica do Nordeste brasileiro (Rylands et al. 2009). São animais de pequeno porte com peso entre 350 e 450 gramas, pelagem cinza claro, estriada nas orelhas e com uma mancha branca na região frontal da cabeça. A cauda é maior do que o corpo e tem a função de garantir o equilíbrio do animal, aspecto importante devido ao seu modo de vida arborícola. Essa espécie alimenta-se de insetos, aranhas, pequenos vertebrados, ovos de pássaros, frutos e são também gumívoros, ou seja, alimentam-se da goma exsudada de troncos de árvores gumíferas. Esta goma serve de fonte de carboidratos, cálcio e algumas proteínas. Os saguis dispendem cerca de 25 a 30\% de seu tempo ativo, procurando por alimentos (Marroig et al. 2004). Formam grupos de 2 a 13 indivíduos, normalmente com uma fêmea reprodutora. 0 cuidado da prole principalmente por machos adultos é uma característica social, sendo considerado como uma estratégia da fêmea reprodutora para haver menos gasto de energia e consequentemente garantir sucesso reprodutivo (Stevenson \& Rylands 1988, Rylands 1989).

Essa espécie de sagui é muito comum e de fácil adaptação ao cativeiro, aspecto que estimula a captura clandestina desses animais. 0 Centro de Triagem de Animais Silvestres do Instituto Brasileiro do Meio Ambiente e dos Recursos Naturais Renováveis do Estado da Paraíba (CETAS/IBAMA/ $\mathrm{PB}$ ) recebe com frequência saguis apreendidos pelos agentes do Instituto Brasileiro do Meio Ambiente e dos Recursos Naturais Renováveis (IBAMA). Esses animais são oriundos de criadores clandestinos e de traficantes de animais silvestres. Muitos saguis apreendidos eram mantidos em recintos inadequados e muitos chegam ao CETAS/IBAMA/ $\mathrm{PB}$ com ferimentos e precisam de cuidados terapêuticos e cirúrgicos, entretanto muitos não sobrevivem.

De acordo com Machin (2007), os animais selvagens em situação de cativeiro para fins de pesquisa ou criação, devem ter a segurança de ser mantidos sem dor, angústia e sofrimento, pois os animais sofrem efeitos deletérios se a dor não for tratada de forma adequada, podendo resultar em mudanças comportamentais, perda de peso, lesões musculares, depressão da função respiratória, aumento da pressão arterial, automutilação e potencial de desenvolvimento de estados de dor crônica.

Na formação dos Médicos Veterinários as disciplinas de anatomia são direcionadas para os animais domésticos e a falta de conhecimento da anatomia de saguis e outros animais silvestres dificulta a aplicação segura de procedimentos clínicos, cirúrgicos e terapêuticos. Pesquisas básicas em primatas não humanos, em especial aquelas que abordam aspectos anatômicos, são de grande valia para Medicina Veterinária e Ciências Biológicas. Desta forma, o trabalho teve por objetivo caracterizar macroscopicamente os nervos do plexo braquial de saguis, identificando suas origens, visando fornecer dados que possam auxiliar futuras pesquisas com essa e com outras espécies de animais.

\section{MATERIAL E MÉTODOS}

Local e animais da pesquisa. 0 estudo foi realizado no Laboratório de Anatomia Veterinária da Unidade Acadêmica de Medi- cina Veterinária do Centro de Saúde e Tecnologia Rural (CSTR) da Universidade Federal de Campina Grande (UFCG).

Foram usados dez cadáveres de saguis-de-tufos-brancos (Callithrix jacchus Linnaeus, 1758) adultos, cinco machos e cinco fêmeas, com diferentes históricos de morte, doados pelo CETAS/ IBAMA/PB ao Laboratório de Anatomia Veterinária da Universidade Federal de Campina Grande (UFCG). Ao chegarem ao Laboratório os cadáveres foram fixados e conservados em solução aquosa de formol a $10 \%$.

Dissecação do plexo braquial. Com o uso de materiais básicos de dissecação e com o auxílio de uma lupa estereoscópica, foi feita uma incisão na linha mediana ventral do tórax para a retirada da pele, do tecido subcutâneo e dos músculos peitorais de ambos os antímeros para expor os vasos e nervos da região axilar e braquial. Em seguida foi retirado o esterno, com incisões nas articulações costocondrais e removidas as vísceras torácicas e cervicais, assim como, o tecido adiposo, para expor parcialmente os corpos vertebrais e os ramos ventrais dos nervos espinhais que originam o plexo braquial de ambos os antímeros. Posteriormente foram retirados os corpos vertebrais, entre C3 e T3 até a exposição completa da medula espinhal e das raízes dos nervos espinhais que formam os nervos do plexo braquial. Os protocolos usados neste estudo foram aprovados pelo Comitê de Ética em Pesquisa no Uso de Animais (CEUA) do CSTR/UFCG, processo № CEUA 153-2014 e pelo Sistema de Autorização e Informação em Biodiversidade (SISBIO) do Instituto Brasileiro do Meio Ambiente e dos Recursos Naturais Renováveis (IBAMA) com o protocolo № 44489-1 em 26 de maio de 2014. Toda nomenclatura adotada foi baseada na Nomenclatura Anatômica Veterinária (International Committee on Veterinary Gross Anatomical Nomenclature 2012).

\section{RESULTADOS}

Foi observado que em todos os saguis-de-tufos-brancos (Callithrix jacchus) o plexo braquial se origina dos nervos espinhais C5 (nervo cervical 5), C6 (nervo cervical 6), C7 (nervo cervical 7), C8 (nervo cervical 8), T1 (nervo torácico 1) (Fig.1). A formação de cada nervo do plexo está demonstrada na Figura 2. 0 nervo supraescapular originou-se em todos os saguis do nervo espinhal C5, apenas um animal teve contribuição do nervo espinhal C6. Os nervos subescapular, musculocutaneo e axilar originaram-se do nervo espinhal C6 em seis saguis, nos outros saguis os referidos nervos tiveram a origem e participação dos nervos espinhais C5, C6 e C7. 0 nervo radial originou-se em todos os saguis no nervo espinhal $\mathrm{C} 7$, em sete animais houve a contribuição do C8 e um animal teve contribuição de C6. Na formação do nervo mediano participaram os nervos espinhais C6, C7, C8 e T1, sendo que o último contribuiu apenas em um animal. Os nervos ulnar e toracodorsal originaram-se dos nervos espinhais C8 e T1 em seis dos casos e nos outros houve variações na origem e contribuições dos nervos C6, C7, C8 e T1.

Nos dez saguis do presente trabalho, o plexo foi formado a partir de três troncos: tronco cranial, composto pelos nervos supraescapular, subescapular, musculocutâneo, mediano e axilar, sendo o nervo supraescapular exclusivo do tronco. 0 tronco médio, constituído pelos nervos subescapular, musculocutâneo, mediano, radial, axilar e o toracodorsal, e o tronco caudal formado pelos nervos mediano, ulnar, radial e toracodorsal, sendo apenas o nervo ulnar restrito a este tronco (Quadro 1). 


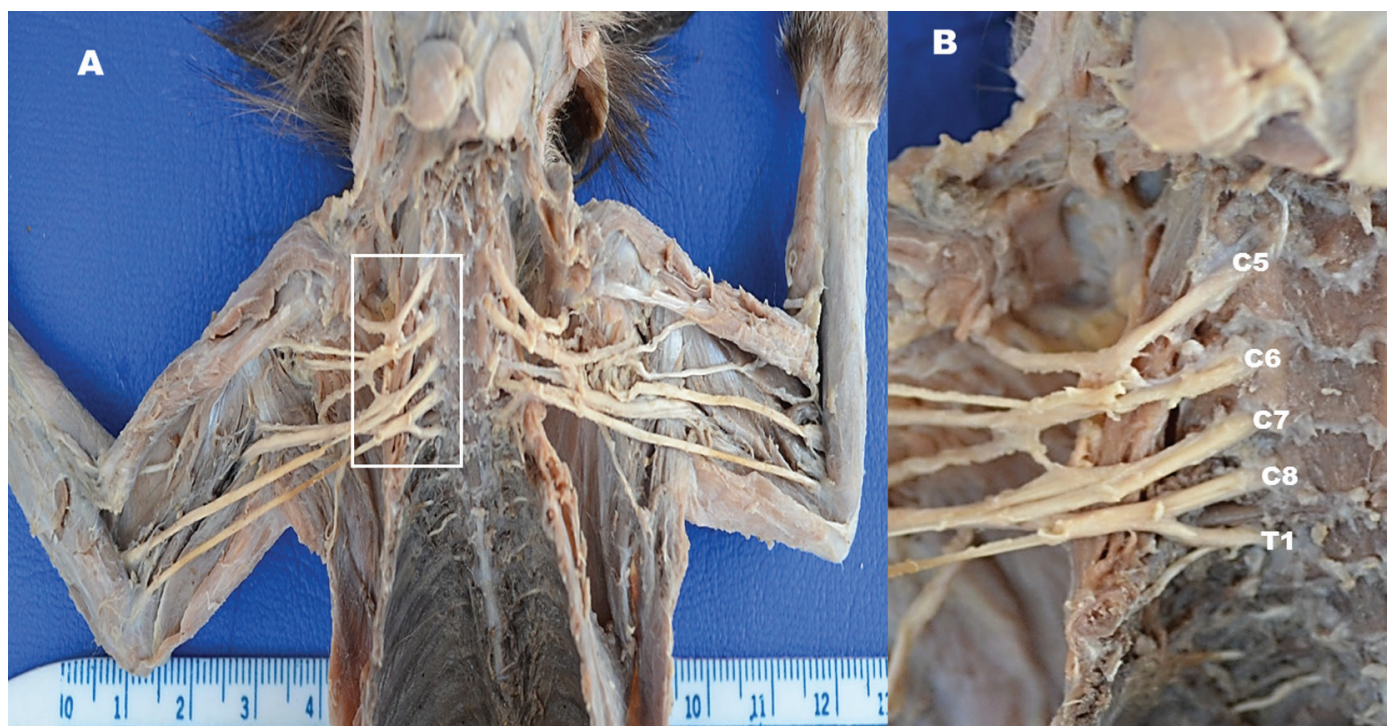

Fig.1. (A) Vista ventral do plexo braquial do sagui-de-tufos-brancos (Callithrix jacchus). Foram removidas as vísceras torácicas e cervicais. (B) Imagem ampliada do quadro da Figura A demonstrando a origem do plexo braquial. Nervo cervical 5 (C5), nervo cervical 6 (C6), nervo cervical 7 (C7), nervo cervical 8 (C8), nervo torácico 1 (T1).

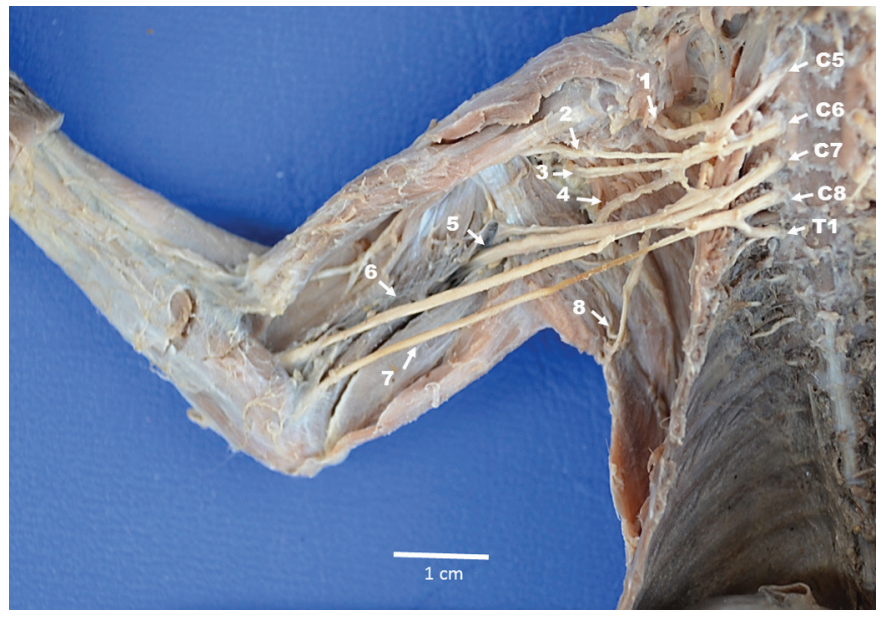

Fig.2. Vista ventral do plexo braquial do sagui-de-tufos-brancos (Callithrix jacchus). Nervo supraescapular (1), nervo musculocutâneo (2), nervo axilar (3), nervo subescapular (4), nervo radial (5), nervo mediano (6), nervos ulnar (7), nervo toracodorsal (8). Nervo cervical 5 (C5), nervo cervical 6 (C6), nervo cervical 7 (C7), nervo cervical 8 (C8), nervo torácico 1 (T1).

\section{Quadro 1. Origem e nervos que constituem o plexo braquial} de Callithrix jacchus

\begin{tabular}{ll}
\hline Troncos & \multicolumn{1}{c}{ Nervos } \\
\hline Tronco cranial & $\begin{array}{l}\text { N. supraescapular, n. subescapular, } \\
\text { n. musculocutânio, n. axilar, n. mediano }\end{array}$ \\
Tronco médio & $\begin{array}{l}\text { N. subescapular, n. musculocutânio, n. axilar, } \\
\text { n.mediano, n. radial, n. toracodorsal }\end{array}$ \\
Tronco caudal & N. radial, n. mediano, n. ulnar, n. toracodorsal
\end{tabular}

\section{DISCUSSÃO}

Colaborando com a anatomia comparativa, bem como, com os padrões de referências para diagnósticos clínicos de lesões de nervos periféricos, ou mesmo de medula, bem como com a anestesia do plexo braquial do sagui-de-tufo-branco (Callithrix jacchus), foi observado que a origem e formação do plexo braquial são semelhantes à dos primatas já descritos, como nas espécies do gênero Macaca (Sugiyama 1965), em gálagos (Galago senegalensis senegalensis) (Kanagasuntheram \& Mahran 1960), macaco-prego-de-cara-branca (Cebus capucinus) (Mizuno 1969), gorila (Gorilla sp.), chimpanzés e micos (Troglotydes niger e Cynocephalus anubis) (Champneys 1975), babuínos (Papio ursinus) (Booth et al. 1997), chimpanzé (Pan sp.), orangotango (Pongo sp.), gibão (Hylobates sp.) (Oliveira et al. 2003), macaco-prego (Cebus apella) (Ribeiro et al. 2005), macaco-barrigudo (Lagothrix lagothricha) (Cruz \& Adami 2010), macaco-de-cheiro (Saimiri sciureus) (Araújo et al. 2012). Em primatas são observadas algumas variações na origem do plexo braquial em comparação aos mamíferos domésticos. Essas variações podem ocorrer durante o processo evolutivo, no qual o plexo braquial desloca-se cranialmente, atingindo o nervo C4 nos símios e C3 em humanos (Parada et al. 1989).

A origem do plexo braquial no sagui se estendeu de C5 a T1, como ocorre na maioria dos macacos do Novo Mundo (Sugiyama 1965, Hill 1972). Quanto à origem em C5, o Callithrix jacchus se assemelha ao gálago (Galago senegalensis), babuíno (Papio ursinus), guenon (Cercopithecus), macaco-prego-de-cara-branca (Cebus capucinus) e macaco-barrigudo (Lagothrix sp.). Porém, diferencia-se dos chimpanzés (Pan sp.), gorilas (Gorilla sp.), macaco-de-cheiro (Saimiri sciureus), Macaca (Macacus cyclopsis), macaco-prego (Cebus apella) e orangotango (Pongo sp.) que começa em C4. Tais diferenças são, de certo modo, sem expressividade funcional aparente, apesar dos Callithrix apresentarem um hábito de escalado diferente dos primatas de porte maior, como os Macacus e os Cebus, por serem menores, tendo que escalar com os membros em maior grau de extensão, seus movimentos de apoio poderiam, durante o processo evolutivo, acarretar no deslocamento mais cranial dos segmentos que compõem a inervação do membro torácico, o que não é comprovado neste estudo, necessitando de mais pesquisas para tal. 
Nos saguis também foi sugerido usar a nomenclatura baseada em apenas três troncos, o cranial formado por C5 e C6, o médio formado por C7 e o caudal formado por C8 e T1 (ocasionalmente com participação de T2). Tal situação é bastante diferente no macaco-de-cheiro (Saimiri sciureus) (Araújo et al. 2012) devido à participação direta de C4, onde há formação de quatro troncos, com localizações bem precisas, sendo o primeiro (cranial) em C4, o segundo (médio-cranial) em C5 e C6, o terceiro (médio-caudal) em C7 e C8 e o quarto (caudal) em T1. Essa nomenclatura utilizada, comumente em humanos, vem sendo adotada também em primatas não humanos (Araújo et al. 2012), o que facilita a identificação de grupos de nervos da região axilar, colaborando com o estudo da espécie, bem como com a clínica cirúrgica nesta e em outras espécies de pequenos primatas.

\section{CONCLUSÃO}

O plexo braquial de Callithrix jacchus mostrou-se constituído de nervos que são originados de segmentos medulares C5 a T1, com padrão de formação semelhante à maioria dos primatas já estudados, mostrando, para esta espécie, potencial no uso de técnicas diagnósticas, anestésicas e cirúrgicas já descritas para primatas de outros gêneros com semelhanças anatômicas à Callithrix.

\section{REFERÊNCIAS}

Araújo E.B., Lima A.R., Pinheiro L.L., Muniz J.A.P.C., Imbeloni A. \& Branco E. 2012. Origem do plexo braquial de Saimiri sciureus. Pesq. Vet. Bras., 32(12):1351-1354.

Booth K.K., Baloyi F.M. \& Lukhele O.M. 1997. The brachial plexus in the Chacma baboon (Papio ursinus). J. Med. Primatol. 26:196-203.

Champneys F. 1975. On the muscles and nerve of a Chimpanzee (Troglodytes niger) and Cynocephalus anubis. J. Anat. Physiol. 6(1):176-211.

Cruz G.A.M. \& Adami M. 2010. Anatomia do plexo braquial de macaco-barrigudo (Lagothrix lagothricha). Pesq. Vet. Bras. 30(10):881-886.

Hill W.C.O. 1972. Primates: comparative anatomy and taxonomy. V. Cebidae (part B). Edinburgh University Press, Edinburgh. 537p.
International Committee on Veterinary Gross Anatomical Nomenclature 2012. Nomina Anatomica Veterinaria. 5th ed. Hannover. 166p.

Kanagasuntheram R. \& Mahran Z.Y. 1960. Observations on the nervous system of the lesser bush baby (Galago senegalensis senegalensis). J. Anat. 94(4):512-527.

Machin K.L. 2007. Wildlife analgesia, p.43-60. In.: West G., Heard D. \& Caulkett N. (Eds), Zoo Animal and Wildlife Immobilization and Anesthesia. Blackwell Publishing, Ames.

Marroig G., Cropp S. \& Cheverud J.M. 2004. Systematics and evolution of the jacchus group of marmosets (Platyrrhini). Am. J. Phys. Anthropol. 123:11-22.

Mizuno N. 1969. The brachial plexus in the Squirrel Monkey (Saimiri sciureus). Primates 10:19-35.

Oliveira M.F., Miglino M.A., Passipieri M., Teixeira D.G., Moura C.E.B., Martins D.S., Benedicto H., Thomaz J.M. \& Ambrósio C.E. 2003. Anatomia do plexo braquial do macaco Gibão (Hylobates sp.). Int. J. Morphol. 21(1):59.

Parada H., Pineda U.H., Lagunas E.M. \& Vidal H.A. 1989. Variaciones anatómicas de las ramas raquídeas que constituyen los troncos de origen del plexo braquial. Anales Anat. Normal 7:32-36. (Apud Moura et al. 2007)

Ribeiro A.R., Prada I.L.S., Silva Z., Barros R.A.C. \& Silva D.C.O. 2005. Origem do plexo braquial do macaco Cebus apella. Braz. J. Vet. Res. Anim. Sci. 42(2):143-149.

Rylands A.B. 1989. Evolução do sistema de acasalamento em Callitrichidae, p.87-108. In: Ades C. (Ed.), Etologia de Animais e de Homens. Edicon/Edusp, São Paulo.

Rylands A.B., Coimbra-Filho A.C. \& Mittermier R.A. 2009. The systematics and distributions of the marmosets (Callithrix, Callibella, Cebuella and Mico) and Callimico (Callithichidae, Primates), p.25-61. In: Ford S.M., Porter L.M. \& Davis L.C. (Eds), The Smallest Anthropoids, Developments in Primatology: progress and prospects. Springer, London.

Rylands A.B. 2012. Taxonomy of the Neotropical Primates: database. International Union for Conservation of Nature (IUCN), Species Survival Commission (SSC), Primate Specialist Group, Gland.

Stevenson M.F. \& Rylands A.B. 1988. The marmosets, genus Callithrix, p.131-222. In: Mittermeier R.A. \& Coimbra Filho A.F. (Eds), Ecology and Behavior of Neotropical Primates. Vol. 2. World Wildlife Fund, Washington D.C.

Sugiyama T. 1965. On the plexus brachialis of Macacus cyclopsis. Acta Med. Nagasaki 9(3):65-68. 